\title{
Hydrodynamic drainage force in a highly confined geometry: role of surface roughness on different length scales
}

\author{
S. Guriyanova $\cdot$ B. Semin $\cdot$ T. S. Rodrigues $\cdot$ \\ H.-J. Butt · E. Bonaccurso
}

Received: 29 May 2009/Accepted: 18 August 2009/Published online: 1 October 2009

(c) The Author(s) 2009. This article is published with open access at Springerlink.com

\begin{abstract}
We measured the hydrodynamic drainage force of an aqueous, Newtonian liquid squeezed between two hydrophobic or two hydrophilic surfaces by means of the colloidal probe technique. We controlled the wettability, the roughness, the topology, and also the approaching velocity of the surfaces. We found that asperities on the surfaces caused an artificial decrease of the measured drainage force that must be considered by the interpretation of the force curves. Even considering the effect of asperities, our experimental results could be interpreted only with the aid of a partial slip model. Or else, interpreted assuming that the viscosity close to the surfaces is different from bulk. On patterned hydrophilic surfaces, we demonstrated that the drainage force depends not only on the overall surface roughness or micro structuring but also on the specific length scale of the surface nanostructures.
\end{abstract}

Keywords Colloidal probe technique .

Slip boundary condition - Hydrodynamic drainage force . Nanoscopic roughness

\section{Introduction}

In classical fluid mechanics, it is commonly made the assumption that liquid molecules directly in contact with a solid surface are stationary relative to the solid (Bernoulli 1738; Du Buat 1779; Stokes 1845, 1966). This is the so-called no-slip boundary condition (BC), which has been

S. Guriyanova - B. Semin - T. S. Rodrigues - H.-J. Butt ·

E. Bonaccurso $(\square)$

Max Planck Institute for Polymer Research, Ackermannweg 10, 55128 Mainz, Germany

e-mail: bonaccur@mpip-mainz.mpg.de successfully used over the last centuries for modeling macroscopic fluid flows of most Newtonian and some nonNewtonian liquids. Despite this, recent sensitive measurements have shown that the no-slip BC may break down at the micro- and nanoscale. Under certain circumstances also a Newtonian liquid is allowed to slip along the solid/liquid boundary (for extensive reviews see Vinogradova 1999; Ellis and Thompson 2004; Lauga et al. 2005; Neto et al. 2005). The interest in fluid flows at these small scales has been driven by fast developments in the fields of microfluidics, micro-electro-mechanical systems, and lab-onchip technologies, where water is mostly used as solvent. It also has implications in flows in porous media, particle aggregation, boundary lubrication, and in the vast majority of biological processes.

It is accepted that surface wettability (Pit et al. 2000; Bonaccurso et al. 2002; Choi et al. 2003; Qian et al. 2005; Cottin-Bizonne et al. 2008; Maali et al. 2008), surface roughness (Zhu and Granick 2002; Bonaccurso et al. 2003; Truesdell et al. 2006; Vinogradova and Yakubov 2006), and super hydrophobicity (Bhushan et al. 2009) influence the interaction between liquid and solid at the interface. Thus, the boundary condition necessary for describing the flow of liquid on a solid must be adapted to such situations. On hydrophobic or super hydrophobic surfaces, the interaction has been found to be drastically reduced due to the entrapment of air by the surface asperities. If one uses a slip BC model to describe the fluid flow resulting from such a reduced interaction due to an air cushion, slip lengths from few hundred nanometers up to tens of micrometers have been found (Tretheway and Meinhart 2004; Lee et al. 2008). The presence of lubricating species like hydrated ions at the surface influences the mobility of water (Donose et al. 2005; Guriyanova and Bonaccurso 2008). Also the polarity of the liquid does, since molecules 
with a strong dipole moment are less mobile on surfaces than non-polar ones (Cho et al. 2004). All these factors are strongly linked, and their effects cannot be easily decoupled. This is one reason for the current unresolved debate.

Nearly all of the first reports, and a few of the recent ones, suggested that if slip occurred it was only on nonwetted surfaces (Churaev et al. 1984; Blake 1990; Baudry et al. 2001; Tretheway and Meinhart 2002; Cottin-Bizonne et al. 2008; Maali et al. 2008). However, the occurrence of slip was also shown for partially or totally wettable surfaces (Pit et al. 2000; Craig et al. 2001; Bonaccurso et al. 2002; Sun et al. 2002; Zhu and Granick 2004; Joseph and Tabeling 2005; Willmott and Tallon 2007, 2008). Surface roughness has been theoretically predicted to both increase (Hocking 1976; Baldoni 1996) or decrease the degree of slip (Richardson 1973; Jabbarzadeh et al. 2000; Ponomarev and Meyerovich 2003). On the experimental side, the situation is also unclear. Pit et al. (2000) and Zhu and Granick (2002) showed that in the presence of thin polymer films, which increase the surface roughness, slip was reduced. Bonaccurso et al. (2003) showed that in a completely wetting system, the degree of slip increased as the surface roughness increased. Schmatko et al. (2006) found that the wavelength of the roughness influences slip more than the height of the asperities. For roughness in the range of tens of nanometers, they found slip lengths to be hundreds of nanometers. With respect to the shear rate, some authors found that it influenced the solid-liquid interaction (Brochard and de Gennes 1992; Craig et al. 2001; Zhu and Granick 2001), while others did not (Pit et al. 2000; Bonaccurso et al. 2002; Cottin-Bizonne et al. 2004; CottinBizonne et al. 2008).

For different techniques, the measured slip lengths are within experimental error, meaning that one cannot distinguish between a slip and a no-slip case. Here, we name just a few examples: Cottin-Bizonne et al. found that water did slip on hydrophobized surfaces, but not on bare hydrophilic Pyrex glass. They used a surface force apparatus type and their experimental error was $\pm 2 \mathrm{~nm}$ (CottinBizonne et al. 2008). Honig and Ducker used the colloidal probe technique with stiffer cantilevers than other groups, and they found no slip of liquids on wetted surfaces. Their experimental error was $\pm 5 \mathrm{~nm}$ (Honig and Ducker 2007, 2008). Willmott and Tallon found rather large slip lengths of up to $75 \mathrm{~nm}$ using a torsional ultrasonic oscillator, for both hydrophobic and hydrophilic surfaces. Their experimental error, however, was up to $\pm 100 \mathrm{~nm}$ (Willmott and Tallon 2007, 2008).

Vinogradova and colleagues (Fan and Vinogradova 2005; Vinogradova and Yakubov 2006) showed theoretically and experimentally that slippage or the existence of asperities provide pressure relief. Correspondingly they will reduce the total drainage force in a hydrodynamic measurement with the colloidal probe technique. The pressure is locally determined by the size and the shape of the asperities. Simulated force curves for single asperities with different radii of curvature led them to predict that an asperity with a smaller radius caused a stronger reduction of the drainage force. An experiment with a roughened colloidal probe corroborated the prediction. The separation was measured between the flat surface and the closest asperity on the colloidal probe. The latest results by Vinogradova led us to study the influence of size (height and radius of curvature) of asperities on the hydrodynamic drainage force and also to perform a whole series of experiments on many different surfaces and with many different colloidal probes. We measured the hydrodynamic force between hydrophobic and hydrophilic surfaces (flats and colloidal probes), having different surface topologies and roughness on the micrometer and on the nanometer scale.

\section{Methods}

The experiments were performed on an atomic force microscope (AFM) (MultiMode PicoForce in closed-loop scanning mode, Veeco Instruments, Santa Barbara, CA) using the colloidal probe technique (CPT) (Butt 1991; Ducker et al. 1991) This technique measures the drainage force of a liquid squeezed between a hard, flat surface and an approaching sphere. Borosilicate glass spheres (Duke Scientific Corp., Palo Alto, CA) of radius $R=9.6 \pm 1 \mu \mathrm{m}$ were glued to the free end of tipless, V-shaped, silicon nitride cantilevers (Veeco Instruments, Santa Barbara, CA; nominal spring constant $k_{\mathrm{c}}=0.06 \mathrm{~N} / \mathrm{m}$ ). True spring constants were measured by the thermal noise method (Hutter and Bechhoefer 1993; Butt and Jaschke 1995) and are given separately for each experiment. The error in this method is between 5 and 10\% (Matei et al. 2006; Ohler 2007). The radii $R$ of the colloidal probes were determined from scanning electron microscopy (SEM) images (LEO 1530 Gemini, Zeiss-LEO GmbH, Oberkochen, Germany). We did this by fitting a circle to at least two different views of a particle. The error was around $2 \%$. For clarity of presentation, we only indicate the average values of particle radii and spring constants, without specifying errors.

A batch of colloidal probes and silicon samples were cleaned in air plasma at medium power $(30 \mathrm{~W})$ for $30 \mathrm{~s}$ (PDC-002, Harrick Scientific Inc., Pleasantville, NY) to make them hydrophilic. Another batch of colloidal probes and silicon samples were functionalized by a self-assembled monolayer (SAM) of an alkane thiol ( $n$-dodecyl mercaptan $\left./ \mathrm{CH}_{3}-\left(\mathrm{CH}_{2}\right)_{11}-\mathrm{SH}\right)$ (Sigma-Aldrich Chemie $\mathrm{GmbH}$, München, Germany) to make them hydrophobic. Before the thiolation, surfaces were plasma cleaned, then 
coated with $5 \mathrm{~nm}$ of chromium (as an adhesion promoting layer) followed by $50 \mathrm{~nm}$ of gold by means of thermal evaporation, at an evaporation rate of $0.2 \mathrm{~nm} / \mathrm{s}$ and a pressure of $2 \times 10^{-5}$ mbar (MED-20, BAL-TEC AG, Liechtenstein). Thereafter, the samples were immersed in a $1 \mathrm{mM}$ solution of alkane thiol in ethanol at room temperature for $24 \mathrm{~h}$. After removal from the solution, the probes were rinsed thoroughly with ethanol. Colloidal probes and surfaces were used for the measurements immediately after the preparation. Some planar silicon substrates where used for quality control of the surface modifications by sessile drop contact angle measurements (OCA35, Dataphysics
Instruments, Filderstadt, Germany). The advancing contact angle was about $105^{\circ}$ for the thiolated samples, and $<5^{\circ}$ for the plasma-cleaned samples.

Samples with microscopic and nanoscopic patterns were produced from a silicon (111) wafer using a focused ion beam (FIB) (Raith Elphy Plus, Raith GmbH, Dortmund, Germany). Parallel grooves of controlled depths were etched into the silicon at defined intervals, on $10 \times 10 \mu \mathrm{m}^{2}$ areas. Eight different patterned areas with heights between 15 and $40 \mathrm{~nm}$ and wavelengths of around 400 and $500 \mathrm{~nm}$ were etched on the silicon wafer, next to each other (Fig. 1). This allowed for force experiments on the
Fig. 1 AFM topography images of eight silicon surfaces patterned on different length and height scales. Scale bars are all $1 \mu \mathrm{m}$

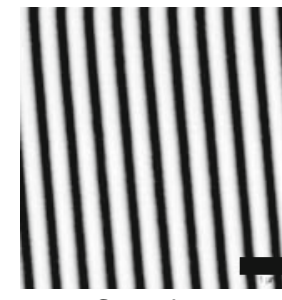

Sample 1

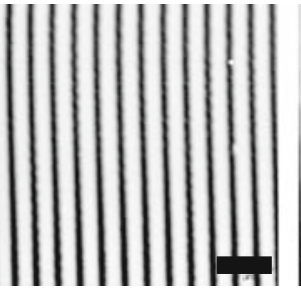

Sample 5
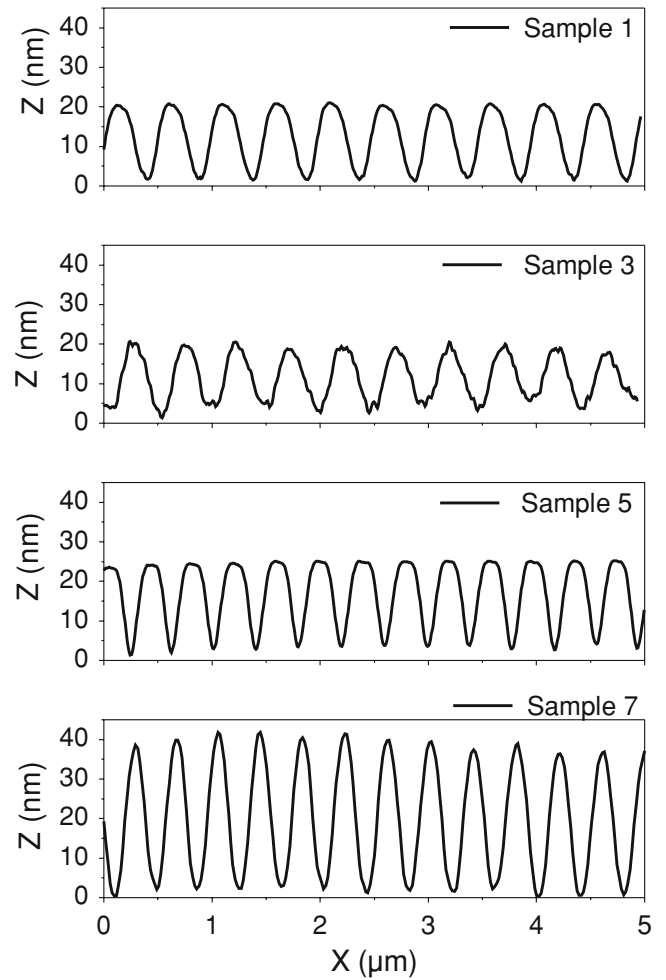

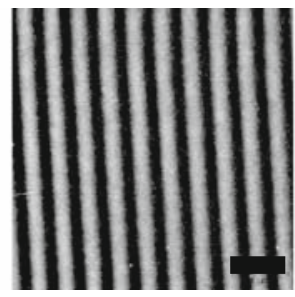

Sample 3

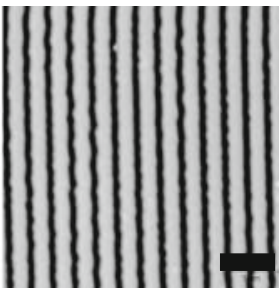

Sample 4

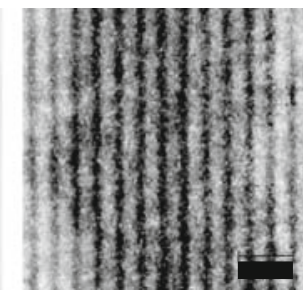

Sample 8

Sample 7
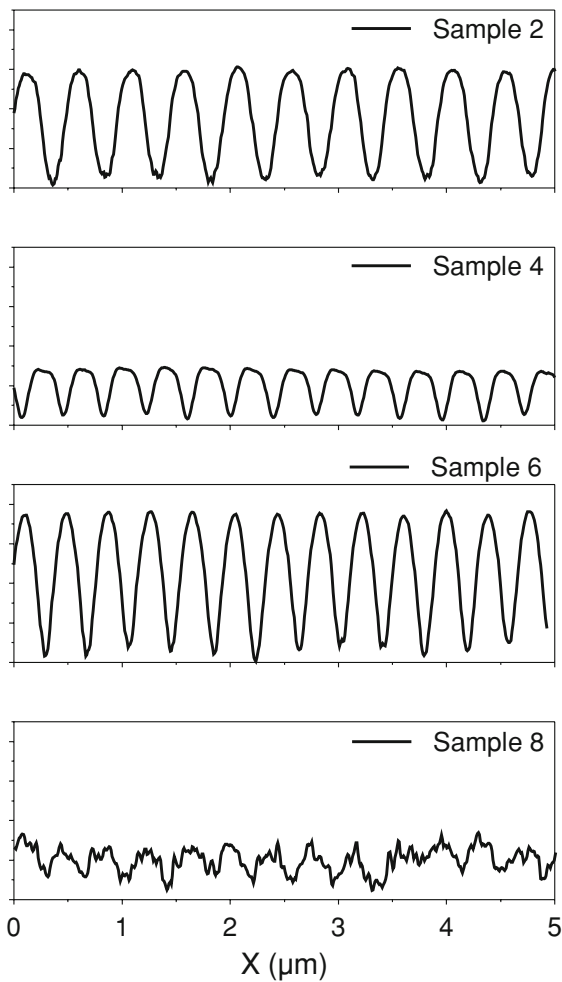
different areas and on the smooth wafer with the same colloidal probe without the need of opening of the AFM liquid cell in between the measurements. The section of the ripples was sinusoidal (Samples 1, 2, 6, and 7, and Fig. 2a), sinusoidal with a superimposed nanometer-scale roughness (Samples 3 and 8, and Fig. 2b), or sinusoidal with a flattened top (Samples 4 and 5). The samples with the superimposed nanometer-scale roughness were fabricated using a very large beam power, which caused a re-deposition of material on the ripples. The flat-top samples were generated in two steps, first etching the sinusoidal patterns and later removing the top of the ripples by ion milling.

Samples functionalized by thiolation and those patterned by FIB were imaged in air with AFM in tapping mode for determining the surface roughness (root-mean-squareRMS, and peak-to-valley-PV). The colloidal probes were "reverse imaged" in contact mode in air by employing a standard calibration grating (TGT01, NT-MDT Co., Moscow, Russia) according to a well-established technique (Neto and Craig 2001). From reverse imaging, we also determined the radius of curvature of the colloidal probes. It agreed within 5\% with the radius measured by SEM.

Before beginning the force measurement, the sample was mounted onto the piezoelectric AFM scanner. Then, the AFM head with the liquid cell without O-ring and with the colloidal probe were mounted and the liquid cell was filled with the electrolyte solution. The solution remains in the liquid cell due to capillary force. The system was allowed to equilibrate with the laser turned on. The temperature in the cell was around $28 \pm 1{ }^{\circ} \mathrm{C}$, which was $4^{\circ} \mathrm{C}$ above room temperature due to the heating caused by the laser and the AFM electronics. The temperature was monitored using a thermocouple placed close to the colloidal probe. The uncertainty of $\pm 1^{\circ} \mathrm{C}$ in controlling the temperature results in an uncertainty of around 5\% in determining the viscosity of the solution. During a force measurement, the sample was moved up and down at constant velocity $v_{0}$ by applying a voltage to the piezoelectric translator. At the same time, the cantilever deflection was measured. The result of such a measurement is a plot of cantilever deflection versus position of the piezo. From this measurement set, a force-versus-distance curve, briefly called "force curve," was calculated. This is done by multiplying the cantilever deflection with the spring constant to obtain the force, and subtracting the cantilever deflection from the position of the piezo to obtain the distance.

Basically, two types of force curves were performed:

(i) "static measurements," at low approaching/retracting velocity $(0.2$ or $0.4 \mu \mathrm{m} / \mathrm{s})$, for determining the surface forces and

(ii) "dynamic measurements," at high approaching/ retracting velocity ( 35 and $70 \mu \mathrm{m} / \mathrm{s}$ ), for determining the hydrodynamic drainage force between the surfaces.

To rule out the effect of inhomogeneities on the planar substrate, series of about 20 force curves were acquired on different positions on the sample for each driving velocity. In the graphs, we show all measured force curves plotted together. This allows for a visual averaging and estimation of the noise level.

Measurements were performed in $100 \mathrm{mM}$ aqueous solutions of $\mathrm{KCl}$ or $\mathrm{KNO}_{3}$ (AnalaR grade, Sigma-Aldrich Chemie GmbH, Munich, Germany) to shield the long-range electrostatic interaction. Solutions were prepared using Milli-Q water (Milli-Q Gradient, Millipore Corp., Billerica, MA). All solutions were prepared from the respective. The viscosity of the solution was $0.93 \pm 0.02 \mathrm{mPa} \cdot \mathrm{s}$ at a temperature of $28 \pm 1^{\circ} \mathrm{C}$. We determined this by temperaturecontrolled rheological measurements with a double Couette wall rheometer (ARES, Rheometric Scientific GmbH, München, Germany).

We also analyzed the low-speed, retracting force curves to measure the adhesion force $F_{\text {ad }}$ for an additional proof of the surface wettability or cleanliness. Low adhesion $\left(0<F_{\text {ad }} / R<0.05 \mathrm{mN} / \mathrm{m}\right.$, with $R$ the radius of the colloidal probe) is expected if clean, hydrophilic surfaces interact across water. In contrast, the so-called hydrophobic interaction acting between hydrophobic surfaces in water
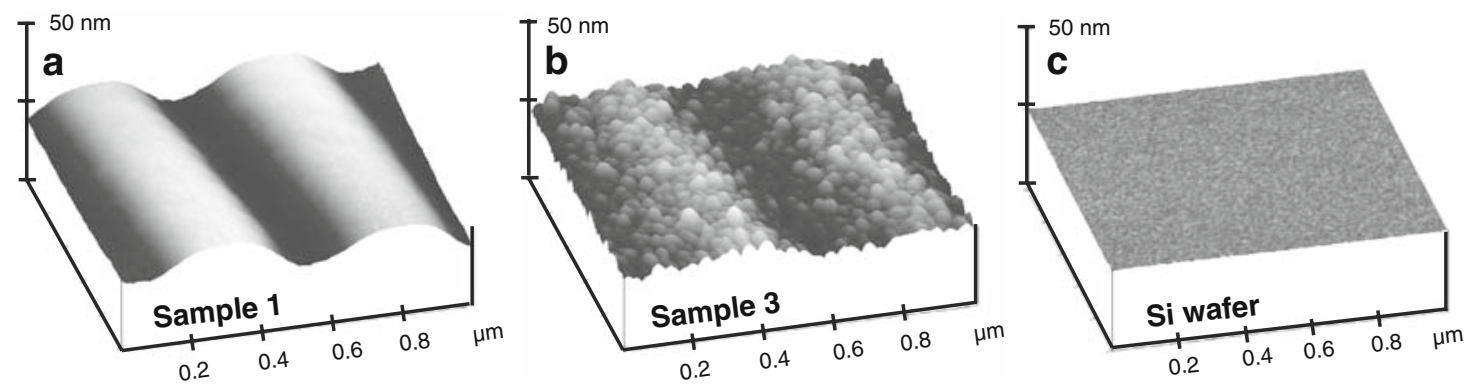

Fig. 2 AFM topography images of two patterned silicon surfaces (samples 1 and 3), and of a smooth silicon wafer 
leads to a stronger adhesion $\left(F_{\text {ad }} / R>4 \mathrm{mN} / \mathrm{m}\right)$. Only the results of sphere-surface systems that passed this test were further evaluated.

\section{Force curve analysis}

We fitted the experimental hydrodynamic force curves with model calculations. Hydrodynamic force curves are calculated solving the equation of motion for a sphere moving toward, or away from, a plane in a fluid:

$F_{\mathrm{h}}+F_{\mathrm{vdW}}+F_{\mathrm{es}}+F_{\mathrm{drag}}+F_{\mathrm{cl}}=m \cdot \frac{\mathrm{d}^{2} h}{\mathrm{~d} t^{2}}$.

Here, $h$ is the separation between sphere and flat surface, $F_{\mathrm{h}}$ is the hydrodynamic force, $F_{\mathrm{vdW}}$ is the van der Waals attraction, $F_{\text {es }}$ is the electrostatic repulsion, $F_{\text {drag }}$ is the hydrodynamic drag on the cantilever, $F_{\mathrm{cl}}$ is the restoring force of the cantilever, and $m \cdot \mathrm{d}^{2} h / \mathrm{d} t^{2}$ takes a possible contribution of the acceleration of the colloidal probe into account. Since our system is characterized by small Reynolds numbers $(\operatorname{Re} \ll 1)$, the acceleration term can be neglected.

For further analysis and discussion of all other terms, please refer to Guriyanova and Bonaccurso (2008). In this article, we refined the analysis concerning the drag on the cantilever, and thus report only on that term.

The viscous drag $F_{\text {drag }}$ on the cantilever increases nearly linearly with the velocity of the cantilever. If the radius of the sphere is large enough, which for purposes means approximately $R>8 \mu \mathrm{m}$, the drag on the cantilever can be considered as a constant contribution (Vinogradova et al. 2001). However, the velocity of the cantilever is not uniform during the approach to the surface: The fixed end of cantilever moves with a constant velocity $v_{0}$, which is the velocity imposed by the piezo, over the whole separation range, while the free end with the glued sphere moves with a velocity $\mathrm{d} h / \mathrm{d} t$ (Fig. 3). This velocity decreases from $v_{0}$, at large separation, to zero, at contact of the sphere with the surface (Fig. 4). Therefore, we take $F_{\text {drag }}$ as non-constant and express it simply using an empirical parameter:

$$
\begin{aligned}
F_{\text {drag }} & =\alpha\left(1-\frac{v_{0}-\mathrm{d} h / \mathrm{d} t}{v_{0}}\right) F_{\mathrm{drag}}^{0}+(1-\alpha) F_{\mathrm{drag}}^{0} \\
& =\left(\frac{v_{0}-\alpha v_{0}-\alpha \mathrm{d} h / \mathrm{d} t}{v_{0}}\right) F_{\mathrm{drag}}^{0}
\end{aligned}
$$

$F_{\text {drag }}^{0}$ is the drag force on a cantilever at large separations, where the whole cantilever moves with constant velocity $v_{0}$, and the parameter $\alpha$ is an empirical correction factor, with $0 \leq \alpha \leq 1$. If we set $\alpha=0, F_{\text {drag }}=F_{\text {drag }}^{0}$, which means that $F_{\text {drag }}$ does not depend on distance. If we set $\alpha=1$, we let the whole cantilever move with the velocity $\mathrm{d} h / \mathrm{d} t$, and then $F_{\text {drag }}$ is underestimated. We found that a

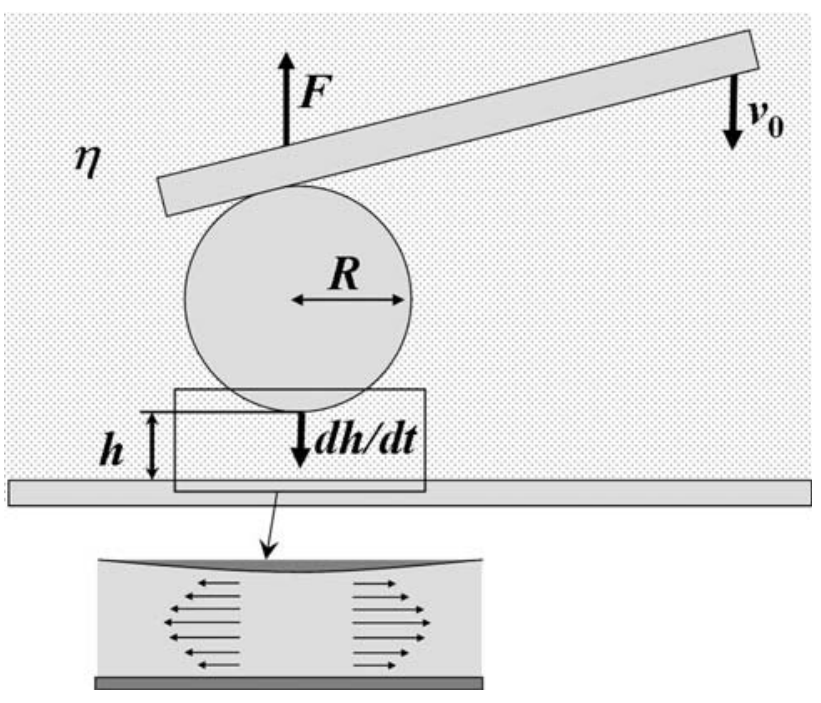

Fig. 3 Scheme of the colloidal probe pushed against a planar surface

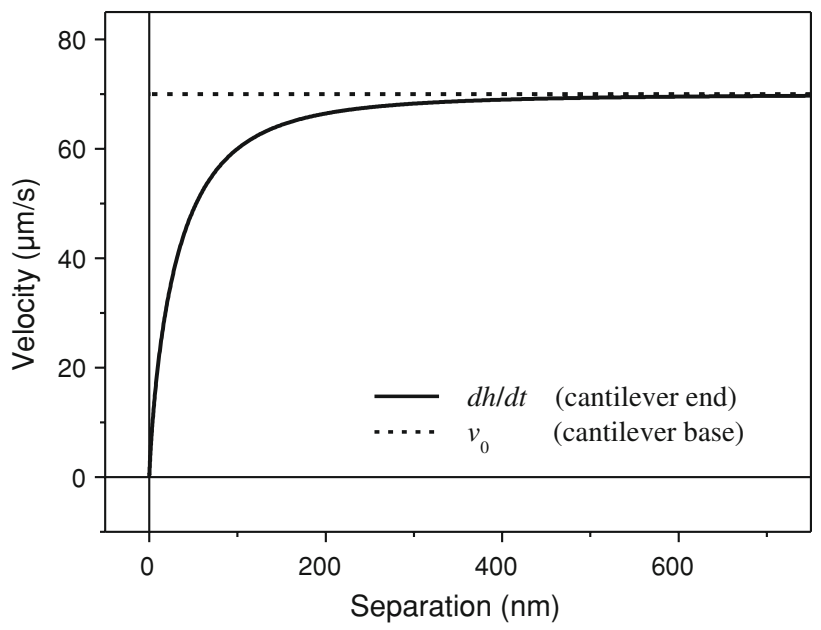

Fig. 4 Calculated velocities of cantilever base (dashed line) and cantilever end (solid line). Parameters: $v_{0}=70 \mu \mathrm{m} / \mathrm{s}, R=10 \mu \mathrm{m}$, $k_{\mathrm{c}}=0.05 \mathrm{~N} / \mathrm{m}, \eta=0.93 \mathrm{mPa} \cdot \mathrm{s}$, and $\alpha=0.8$

value of $\alpha$ between 0.8 and 0.9 described our experimental curves best. A similar value has also been postulated earlier, but using a more elaborate derivation (Vinogradova et al. 2001).

The slip model is used to describe the flow of the liquid between the colloidal sphere and the flat surface. We adopt one of several possible models, the so-called Vinogradova model. With a no-slip BC the hydrodynamic force on the sphere is (Brenner 1961; Chan and Horn 1985)

$F_{\mathrm{h}}=\frac{6 \pi \eta R^{2}}{h} \frac{\mathrm{d} h}{\mathrm{~d} t}$

where $\eta$ is the viscosity of the liquid. Vinogradova (1995) extended the calculations by introducing a correction factor $f^{*}$ taking surface slippage into account: 
$F_{\mathrm{h}}=-\frac{6 \pi \eta R^{2}}{h} \frac{\mathrm{d} h}{\mathrm{~d} t} \cdot f^{*}$

$f^{*}=\frac{h}{3 b} \cdot\left[\left(1+\frac{h}{6 b}\right) \cdot \ln \left(1+\frac{6 b}{h}\right)-1\right]$

Here, $b$ is the slip length. It is a fitting parameter and is not measurable a priori, while all other parameters in Eqs. 2 and 3 can be independently determined from other measurements. For this particular $f^{*}$ it is assumed that both surfaces show the same slipping behavior.

\section{Results and discussion}

We studied the influence of surface asperities on the interfacial flow of a liquid over a solid surface. As examples, we present three illustrative series of measurements:

(i) drainage force between a hydrophobic flat surface and two hydrophobic colloidal particles

(ii) drainage force between a hydrophilic flat surface and two hydrophilic colloidal particles

In both series, one particle had asperities and the other was relatively smooth

(iii) drainage force between two hydrophilic flat surfaces patterned on different length scales and a smooth hydrophilic colloidal particle

For this third series, we show two representative experimental sets of force curves. We then compare the results with measurements on flat silicon surfaces.

\subsection{Hydrophobic surfaces}

Figure 5a and $\mathrm{b}$ shows representative force curves measured using two hydrophobic colloidal probes with $R_{1}=10.4 \mu \mathrm{m}$ and $R_{2}=11.3 \mu \mathrm{m}$. Three representative velocities were chosen, $v_{0}=0.4,35$, and $70 \mu \mathrm{m} / \mathrm{s}$. We first determine the magnitude of the surface forces by fitting the "slow" force curve, and then determine the magnitude of the hydrodynamic force from the "fast" curves. We compared the experimental fast curves with curves calculated with the no-slip and with the slip BC model. For both colloidal probes, and for both fast velocities, we needed to use a finite slip length to fit the experimental curves. We used $b_{1}=90 \mathrm{~nm}$ for the hydrodynamic force on sphere 1 , and $b_{2}=38 \mathrm{~nm}$ for sphere 2 . We also tried to relate the measured reduced hydrodynamic force to surface asperities instead to boundary slip. Analogous to Vinogradova and Yakubov (2006), we shifted the curves by the size of the highest asperities. The hydrophobized flat substrates used in both experimental series had similar surface roughness of about $0.8 \mathrm{~nm}$ RMS over an area $1 \times 1 \mu \mathrm{m}^{2}$, and maximum PV difference of $2.5 \mathrm{~nm}$. Therefore, these cannot account for the discrepancy. Figure $5 \mathrm{c}$ and $\mathrm{d}$ shows AFM images of the sphere's contact area. We calculated the RMS roughness of the spheres after a flattening of second order of their topography images. The roughness over an area of $1.9 \times 1.9 \mu^{2}$ was $6.8 \mathrm{~nm}$ on sphere 1 and $3.4 \mathrm{~nm}$ on sphere 2. Sphere 1 had large asperities, with a maximum PV difference of about $45 \mathrm{~nm}$, while sphere 2 had a lower PV difference of $15 \mathrm{~nm}$. Figure 5e and $\mathrm{f}$ shows a schematic representation of the contact of a sphere and a plane for the case of large asperities on the sphere, and for the case of a relative smooth sphere surface: The large asperities prevent a full contact of the surfaces, unlike the case of smooth surfaces. The dashed curve in Fig. 5e schematically shows the possible run of the experimental curve in the case of a smooth sphere. The slip length we can fit to the force curve when surface asperities are involved is higher than the slip length we would fit if the surfaces got in full contact, as shown in Fig. 5f. This means that on rough surfaces, a part of the slip is an "apparent" slip due to poor contact between the surfaces. However, to fit the experimental curves, we shift the position of hard contact between the flat surface and sphere 1 by $110 \mathrm{~nm}$, and of sphere 2 by $60 \mathrm{~nm}$. These values are larger than the maximal height of the measured asperities, so that, according to Vinogradova's model, a residual, "true" slip is still present. As briefly mentioned above, the model we use to fit the experimental force curves does not give a physical explanation for the slip. It simply introduces a parameter, the slip length $b$, by which the experimental curves can be made fit to the theoretical ones. The physical origin of the reduced drainage force that we measure is still obscure.

\subsection{Hydrophilic surfaces}

Figure $6 \mathrm{a}$ and $\mathrm{b}$ show representative force curves measured on flat hydrophilic silicon substrates using two hydrophilic glass colloidal probes with $R_{3}=10.3 \mu \mathrm{m}$ and $R_{4}=9.4 \mu \mathrm{m}$. We used the same velocities as before. We fitted the experimental curves by using two finite slip lengths, $b_{3}=15 \mathrm{~nm}$ for the hydrodynamic force on sphere 3 , and $b_{4}=6 \mathrm{~nm}$ for sphere 4 . The RMS roughness of the flat silicon substrate was the same in both cases: $0.3 \mathrm{~nm}$ over an area $1 \times 1 \mu^{2}$, and the maximum PV difference was $0.7 \mathrm{~nm}$. The RMS roughness of the sphere surfaces, calculated over $1.3 \times 1.3 \mu^{2}$ after a flattening of second order, was 2.1 and $0.4 \mathrm{~nm}$ for spheres 3 and 4, respectively. Here, only sphere 3 has some asperities, with a maximum PV roughness of about $11 \mathrm{~nm}$ (Fig. 6c), while sphere 4 has a similar PV roughness as the flat silicon, namely $1.5 \mathrm{~nm}$, as is seen in Fig. 6d and e. As in the case of the hydrophobic surfaces, the presence of asperities on the sphere 
Fig. 5 a, b Approaching force curves (only each fourth point is shown, curve consists of 1,024 points) measured on hydrophobic surfaces at low $(0.4 \mu \mathrm{m} / \mathrm{s})$ and high (35 and $70 \mu \mathrm{m} / \mathrm{s}$ ) velocities in aqueous electrolyte (100 mM KCl). $R_{1}=10.4 \mu \mathrm{m}$ and

$R_{2}=11.3 \mu \mathrm{m}, k_{\mathrm{c} 1}=0.051$ $\mathrm{N} / \mathrm{m}$ and $k_{\mathrm{c} 2}=0.049 \mathrm{~N} / \mathrm{m}$. c, $\mathbf{d}$ AFM images of the contact area of the colloidal probes. e, $\mathbf{f}$ Schematic representation of two approaching surfaces for the case of: e large asperities on one of the surfaces and $\mathbf{f}$ relative smooth surfaces; (filled circle) force curves from $\mathbf{a}$ and $\mathbf{b}$ at $70 \mu \mathrm{m} / \mathrm{s}$ (only each eighth point is shown); the dashed curve in $\mathbf{e}$ shows schematically the possible run of the force curve in the case of a smooth sphere
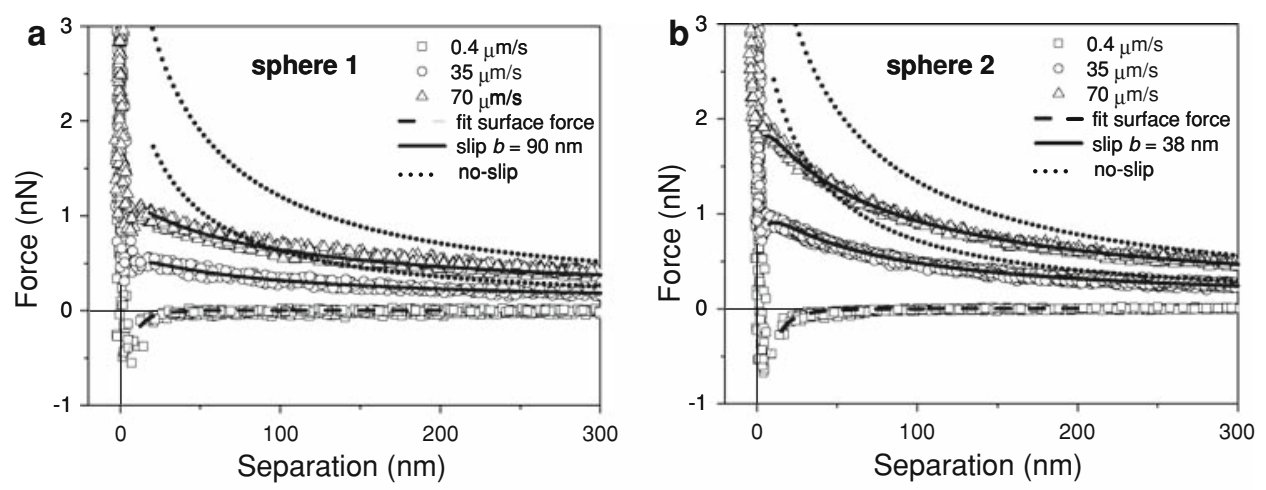

C

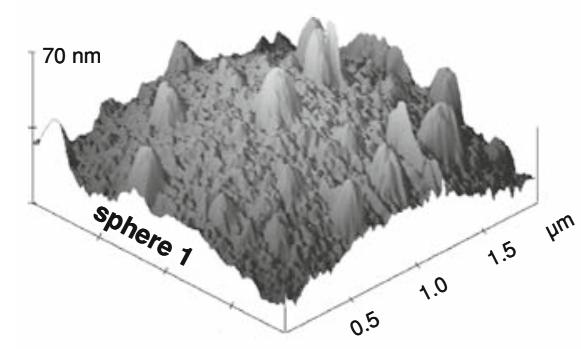

d

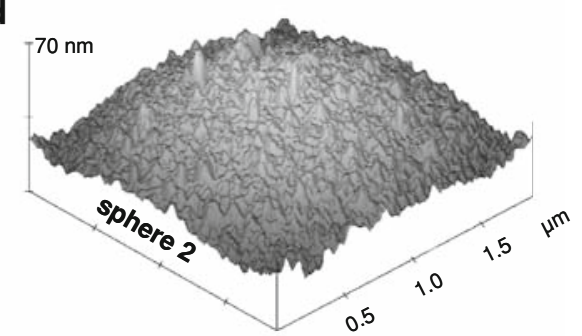

e

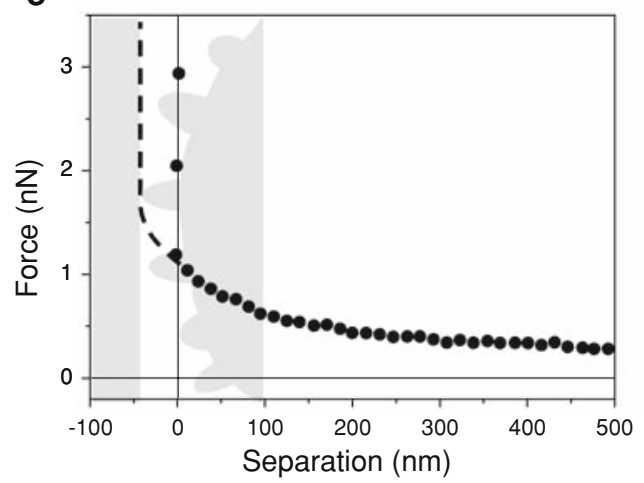

f

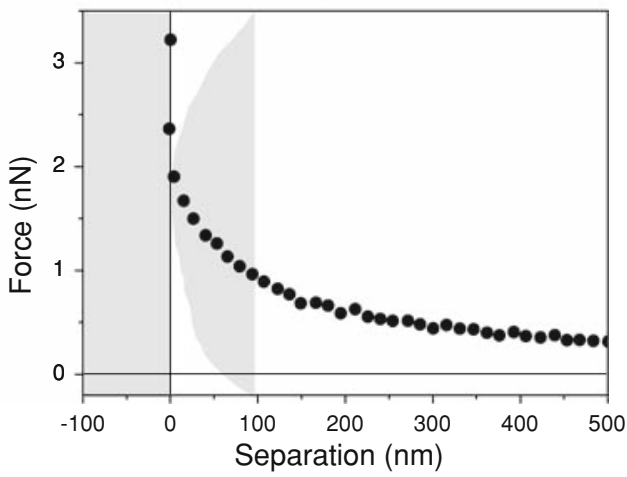

leads to an overestimation of the slip length. If we define zero separation at the base of the asperities of sphere 3, we must shift the position of the solid wall $11 \mathrm{~nm}$ to the right (Fig. 6f). This corresponds to the maximum PV difference on the sphere. Again, we fitted the shifted curves. The best fit using a slip length $b_{3}^{*}=6 \mathrm{~nm}$. This was similar to the value of $b_{4}$ found with the smooth sphere. As above, we conclude that asperities on the sphere prevented a full contact of the surfaces, leading to an "apparent" slip. However, even if we fully account for these asperities, a residual slip occurs according to Vinogradova's model. Therefore, the slip observed on the smooth surfaces could not be explained solely by the surface roughness. We would need to shift the force curves by $9 \mathrm{~nm}$ to fit them with the no-slip model (Fig. 6g), which is larger than the sum of the maximum PV roughness of the sphere and of the sample.

From the results presented above on hydrophobic and hydrophilic randomly rough surfaces, we conclude that we can quantify the reduction of the drainage force by Vinogradova's slip model and slip length $b$. However, we cannot ignore the "apparent" slip induced by asperities on the surface and the sphere. Even taking this into account, a residual "true" slip, whose origin is not yet known, still occurs.

\subsection{Micro- and nanopatterned surfaces}

We present only three drainage curves on patterned surfaces as typical examples for an experimental series on silicon samples with different length scale patterns (Fig. 7). Sample 3 (Fig. 2a) has a regular microscopic roughness, with smooth parallel ripples having a sinusoidal cross section with height around $20 \mathrm{~nm}$ and wavelength around $500 \mathrm{~nm}$. Sample 5 (Fig. 2b) has a similar regular microscopic roughness, but with a superimposed irregular nanoscopic roughness. Cross sections of these surfaces are presented in Fig. 1 and in scale with the colloidal particle also in Fig. 7a. The glass sphere, radius of curvature $R=9.8 \mu \mathrm{m}$, was used for the measurements. From the cross sections, all of comparable $X$ and $Z$ scale, it is evident that during the force measurement, the sphere could 
Fig. 6 a, b Approaching force curves (only each fourth point is shown, curve consists of 1,024 points) measured on hydrophilic surfaces at low $(0.4 \mu \mathrm{m} / \mathrm{s})$ and high ( 35 and $70 \mu \mathrm{m} / \mathrm{s})$ velocities in aqueous electrolyte $(100 \mathrm{mM}$ $\left.\mathrm{KNO}_{3}\right) \cdot R_{3}=10.3 \mu \mathrm{m}$ and $R_{4}=9.4 \mu \mathrm{m}, k_{\mathrm{c} 1}=0.049 \mathrm{~N} / \mathrm{m}$ and $k_{\mathrm{c} 2}=0.052 \mathrm{~N} / \mathrm{m}$. c, d AFM images of the contact area of the colloidal probes. e Zoomed and flattened contact area of sphere 4, having an RMS roughness of $0.3 \mathrm{~nm}$. f, g Force curves acquired at $35 \mu \mathrm{m} / \mathrm{s}$ as shown in $\mathbf{a}, \mathbf{b}$, and the same curves with the contact point shifted by 11 and $9 \mathrm{~nm}$, as represented by the dashed lines
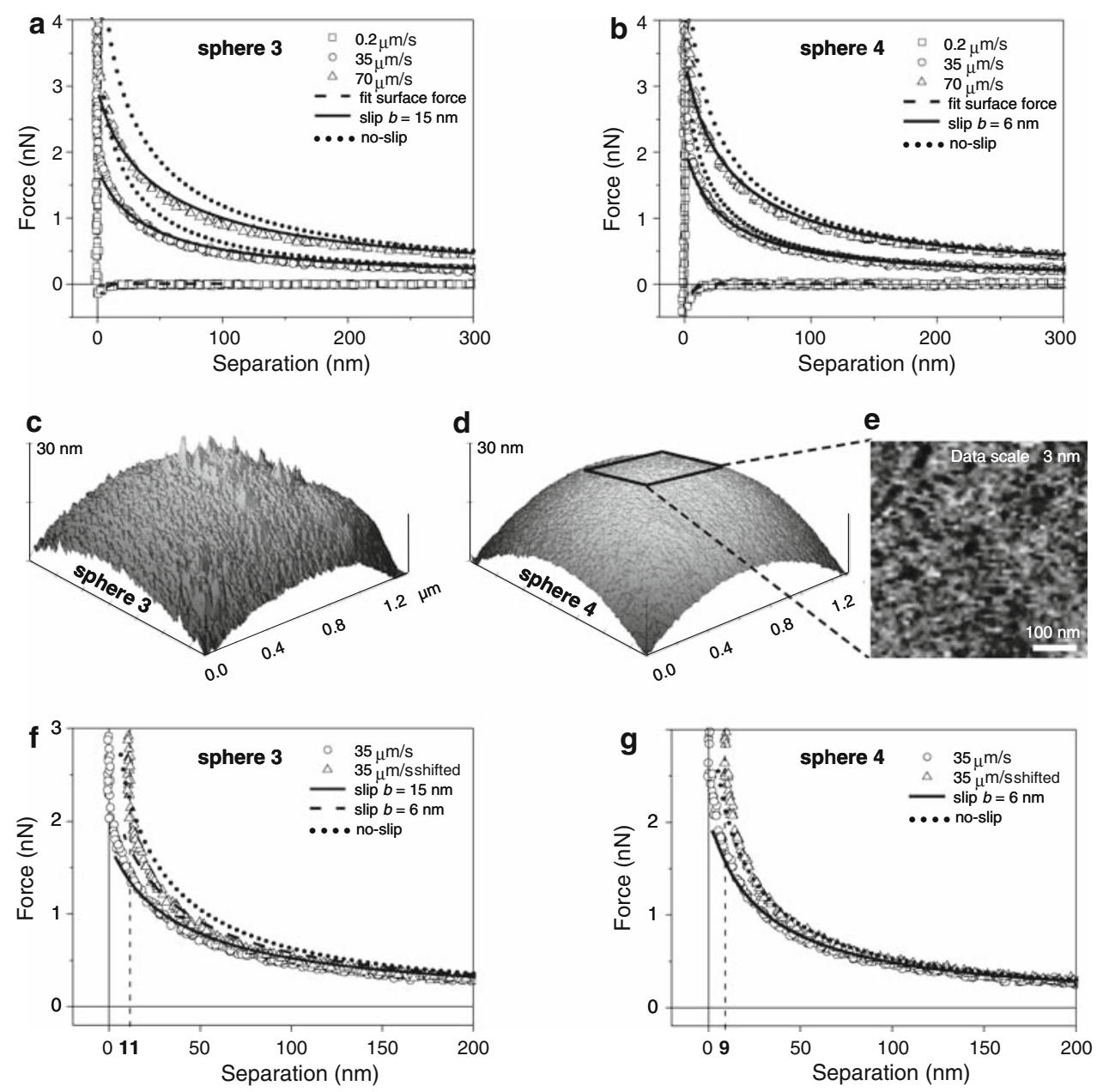

partially fit between the ripples. The third sample (Fig. 2c) was a flat silicon surface without any structure, and was used as the reference surface. The patterned samples' characteristics are presented in Table 1: The wavelength of all the ripples is the same and their height differs by only around $2 \mathrm{~nm}$. The RMS roughness of sample 3 over an area of $0.3 \times 0.3 \mu \mathrm{m}^{2}$ is similar to the one of the smooth silicon wafer $(0.2 \mathrm{~nm})$. The substructure of sample 5 increased the RMS roughness. Because of the small area $\left(10 \times 10 \mu \mathrm{m}^{2}\right)$ of the patterns, we were not able to measure the contact angle of water on them. However, we have observed that the adhesion force measured on the patterned surfaces was similar to adhesion on the flat silicon surface. Therefore, we conclude that the surface energies, and thus the wettabilities, were similar (contact angle $<5^{\circ}$ ).

Figure $7 \mathrm{~b}$ shows representative force curves measured on all three substrates, using the same colloidal probe. It compares curves calculated using the no-slip model to Vinogradova's slip model with slip lengths of 23 and $75 \mathrm{~nm}$. We acquired force curves at different spatial positions, so that in some cases the bottom of the microsphere had contact with a ridge and in others it slotted between two ridges (see Fig. 7a). Please note that the microsphere can penetrate at maximum 3-4 $\mathrm{nm}$ inside the channel between two ridges. In the experimental error limit, we detected no difference between curves acquired on top of the ridges or in between them. The drainage force measured on sample 3 was only slightly lower than the one measured on the smooth surface. In general, the force decreased only slightly for higher asperities compared to the flat silicon surface. In fact, analogous results were obtained for similar surface "smooth" patterns with heights from 10 to $40 \mathrm{~nm}$, wavelengths of 400 and $500 \mathrm{~nm}$, and with other colloidal probes with similar radii of curvature. All these smooth patterns, like samples 1, 2, 4, 5, 6, and 7, give rise to similar drainage forces. These can be described by an apparent slip roughly corresponding to the height of the ripples, and by a residual slip (apparent or real) between 6 and $12 \mathrm{~nm}$.

On the other hand, the "nanorough" patterns, like samples 3 and 8 unexpectedly, give rise to much smaller drainage forces. This is surprising, since the maximum height of the ripples on both the smooth and the nanorough samples is comparable (see profiles in Figs. 1 and 7). The 


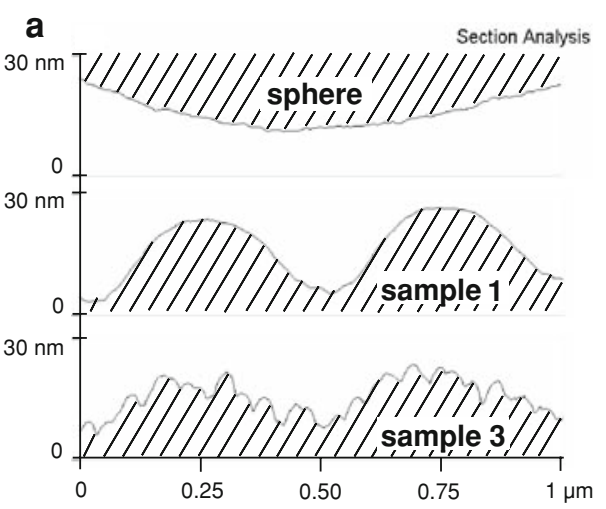

Fig. 7 a Cross section of a sphere with radius $9.8 \mu \mathrm{m}$ and of samples 1 and 3 shown in Fig. 2a and b. b Approaching force curves measured at $70 \mu \mathrm{m} / \mathrm{s}$ in aqueous electrolyte $(100 \mathrm{mM} \mathrm{KCl})$ on the two substrates shown in a and on a flat silicon surface using the same

Table 1 Surface characteristics of samples 1 and 3 shown in Figs. 1, 2 , and 7

\begin{tabular}{|c|c|c|c|c|}
\hline \multirow[t]{2}{*}{ Sample } & \multirow{2}{*}{$\begin{array}{l}\text { Wavelength } \\
(\mathrm{nm})\end{array}$} & \multirow{2}{*}{$\begin{array}{l}\text { Height } \\
(\mathrm{nm})\end{array}$} & \multicolumn{2}{|l|}{ RMS (nm) } \\
\hline & & & $5 \times 5 \mu \mathrm{m}^{2}$ & $0.3 \times 0.3 \mu \mathrm{m}^{2}$ \\
\hline 1 & $\sim 500$ & $\sim 18$ & 6.9 & 0.4 \\
\hline 3 & $\sim 500$ & $\sim 16$ & 4.0 & 1.7 \\
\hline
\end{tabular}

differences of the drainage force could not be equalized by shifting the contact point: the fitted slip lengths differ by around $50 \mathrm{~nm}$. From this we must conclude that the nanoscale asperities are the most probable cause for the reduced drainage force. We therefore partly confirmed Fan's and Vinogradova's predictions (Fan and Vinogradova 2005), namely that the height and the radius of curvature of the patterns, as well as their packing density, determine the interaction between a liquid and a solid surface.

The mechanism for the drag reduction via nanorough features is, however, still unclear. The nanoasperities could act as very effective nucleation sites for gas nanobubbles even on hydrophilic surfaces. They can help trapping the bubbles. An effective carpet of gas bubbles at the liquidsolid interface could reduce the drainage force. It was shown that exceeding a critical flow shear rate, a nanoscopic surface roughness or corrugation can favor the generation of a layer of turbulent flow at the interface. This can modify the viscosity of the fluid in this layer, even if the overall flow is laminar (Prandtl 1927). An example from nature is the so-called shark-skin effect (Bushnell and Moore 1991). Unfortunately, in colloidal probe technique measurements, the shear rate cannot be held constant due to the particular geometry. The shear rate does not only depend on the velocity of approach but also it changes with the separation between the two surfaces. It is not even

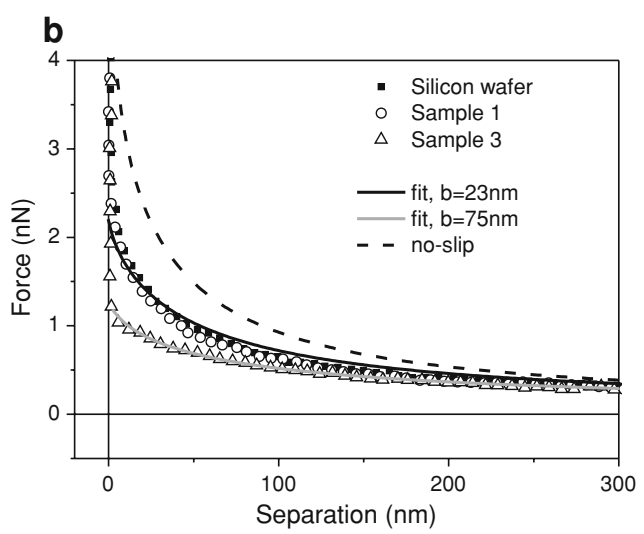

colloidal probe with $R=9.8 \mu \mathrm{m}$. Experimental data (dots) are compared with calculated curves (lines) assuming slip lengths of 0 (dashed line), 23 (black line), and $75 \mathrm{~nm}$ (gray line)

uniform in the same plane (Stark et al. 2006). A critical shear rate could be reached in a single force measurement with colloidal probes. In fact, during a measurement, the shear rate changes from $10^{-4}$ to $10^{4} \mathrm{~s}^{-1}$. Most probably the perturbations are thus created only locally for a short time $(t<50 \mathrm{~ms})$. Similarly, single asperities of nanometer scale have been shown to induce local pressure changes in the liquid flowing past them. They locally change the density of the liquid. Consequently, this induces gradients in the interfacial tension between solid and liquid. These processes have been suggested to generate Marangoni stresses around the asperities, thus disturbing the force balance at the interface and the momentum transfer between liquid and solid (Lukyanov 2009). In Lukyanov's case, though, surface asperities suppress surface slippage. He found the opposite of what we observed.

Of our three experimental series, especially the drainage experiments on the nanoasperities seem to address a key issue. Fitted slip lengths are much larger on nanostructured ripples than on smooth ripples. In literature (Richardson 1973; Jabbarzadeh et al. 2000; Ponomarev and Meyerovich 2003) it is stated that the reduced/enhanced drainage force due to surface rugosity could be an indication that the macroscopic hydrodynamic BCs on a flat and on a patterned surface is different. In those works, an effective no-slip (or stick-slip) BC was applied at some shifted imaginary surface (shear plane). The shear plane for the rough surfaces discussed in the above works was uplifted from the real surface, and thus the slip length was negative. In theory, for some systems, the shear plane could also be shifted inside the surface. This would result in a positive slip length. The authors show that in some cases the no-slip BC can be applied, even if there is actual stick or slip at the rippled surface. In fact, as reported by Ponomarev and Mayerovich (2003), rippled surfaces like ours (samples 1 and 3) should 
Table 2 Summary of experimental results

\begin{tabular}{lcllllc}
\hline Substrate & & & Probe & & $b(\mathrm{~nm})$ & $\begin{array}{c}\text { Curve shift for } \\
\text { no-slip BC (nm) }\end{array}$ \\
\cline { 1 - 2 } \cline { 1 - 1 } Surface type & PV roughness $(\mathrm{nm})$ & & Sphere number & PV roughness $(\mathrm{nm})$ & & \\
\hline Hydrophilic & 1.5 & Sphere 1 & 45 & 90 & 110 \\
& 1.5 & Sphere 2 & 15 & 38 & 60 \\
Hydrophobic & 2 & Sphere 3 & 11 & 15 & 20 \\
& 2 & Sphere 4 & 1.5 & 6 & 9 \\
Sample 1 & 18 & Sphere 5 & 10 & 23 & 45 \\
Sample 3 & 16 & Sphere 5 & 10 & 75 & 85 \\
Flat silicon & 1.5 & Sphere 5 & 10 & 22 & 44 \\
\hline
\end{tabular}

produce higher resistance to the fluid flow. The no-slip BC imposed at the peaks of the ripples would fail, and the shear plane must be further uplifted. This is confirmed by our measurements: the force curves obtained on the flat surface and on the sinusoidal patterns are similar. Our rippled surface thus behaves like a flat surface with the shear plane placed at the top of the ripples. If the BC on both surfaces was similar, however, the shear plane on the rippled surface should be placed at some position between the valleys and the peaks. We thus conclude that the "actual" slip on the rippled surface is smaller as on the flat surface.

Opposite to smooth microscopic ripples that "slow down" the fluid flow, a nanoscale pattern superposed to the sinusoidal microscopic ripples seems to enhance fluid flow. Such a pattern gives rise to more positive slip lengths compared to flat surfaces. Similar results, but limited to a nanoscale pattern only, were found by Zhu and Granick (2002) or by Bonaccurso et al. (2003).

We summarize the results of the three experimental series in Table 2. This clarifies the conclusions we made above. For example, even accounting for surface roughness that is at the origin of some apparent slippage, an additional process causing more (apparent or real) slippage seems to occur. Even if we shifted all curves by the amount of the $\mathrm{PV}$ roughness, we could never recover the no-slip BC. This is demonstrated by the difference between the values of the slip lengths and the shift distances. This finding holds for hydrophobic as well as for hydrophilic surfaces. And much more interestingly, surface roughness at different length scales seems to influence the additional slippage. We find that the transition from "light" to "heavy" slippage takes place for surface features having a radius of curvature between 50 and $400 \mathrm{~nm}$. However, this conclusion could be valid only for the type of sinusoidal ripples described here. Other surface features could change the result.

\section{Summary}

We draw three conclusions from the CPT measurements presented here: (1) The reduction of the drainage force not only depends on the overall roughness but also on the specific surface topology (height, radius of curvature, and packing density of the nano patterns; (2) The effect on interfacial fluid flow is a discontinuous function of the size of the nanopatterns, with a transition between a "light" and a "heavy" slippage; and (3) For the analysis and the interpretation of hydrodynamic force curves, the surface morphology of the sphere and of the sample must be carefully characterized and the presence of single asperities has to be taken in consideration.

Acknowledgments We thank Michael Kappl for programming the force curve data analysis software, Maren Müller and Gunnar Glaßer for the SEM images, Andreas Hanewald for the viscosity measurements, Lars O. Heim, Rüdiger Berger, and Nicole Auth for the preparation of the patterned silicon surfaces, and Mordechai (Rudi) Sokuler for careful proof reading of the manuscript and useful suggestions. We acknowledge financial support from the Max Planck Society (MPG).

Open Access This article is distributed under the terms of the Creative Commons Attribution Noncommercial License which permits any noncommercial use, distribution, and reproduction in any medium, provided the original author(s) and source are credited.

\section{References}

Baldoni F (1996) On slippage induced by surface diffusion. J Eng Math 30:647-659

Baudry J, Charlaix E et al (2001) Experimental evidence for a large slip effect at a nonwetting fluid-solid interface. Langmuir 17:5232

Bernoulli D (1738) Hydrodynamica. Dulsecker, Strasbourg

Bhushan B, Wang Y et al (2009) Boundary slip study on hydrophilic, hydrophobic, and superhydrophobic surfaces with dynamic atomic force microscopy. Langmuir 25:8117-8121. doi:10.1021/ la900612s

Blake TD (1990) Slip between a liquid and a solid: D.M. Tolstoi's (1952) theory reconsidered. Colloids Surf 47:135-145

Bonaccurso E, Kappl M et al (2002) Hydrodynamic force measurements: boundary slip of water on hydrophilic surfaces and electrokinetic effects. Phys Rev Lett 88:076103

Bonaccurso E, Butt H-J et al (2003) Surface roughness and hydrodynamic boundary slip of a Newtonian fluid in a completely wetting system. Phys Rev Lett 90:144501

Brenner H (1961) The slow motion of a sphere through a viscous fluid towards a plane surface. Chem Eng Sci 16:242-251

Brochard F, de Gennes PG (1992) Shear-dependent slippage at a polymer/solid interface. Langmuir 8:3033-3037 
Bushnell DM, Moore KJ (1991) Drag reduction in nature. Annu Rev Fluid Mech 23:65-79

Butt H-J (1991) Measuring electrostatic, van der Waals, and hydration forces in electrolyte solutions with an atomic force microscope. Biophys J 60:1438-1444

Butt H-J, Jaschke M (1995) Calculation of thermal noise in atomic force microscopy. Nanotechnology 6:1-7

Chan DYC, Horn RG (1985) The drainage of thin liquid films between solid surfaces. J Chem Phys 83:5311-5324

Cho J-HJ, Law BM et al (2004) Dipole-dependent slip of Newtonian liquids at smooth solid hydrophobic surfaces. Phys Rev Lett 92:166102

Choi C-H, Westin KJ et al (2003) Apparent slip flows in hydrophilic and hydrophobic microchannels. Phys Fluids 15:2897-2902

Churaev NV, Sobolev VD et al (1984) Slippage of liquids over lyophobic solid-surfaces. J Colloid Interface Sci 97:574-581

Cottin-Bizonne C, Barentin C et al (2004) Dynamics of simple liquids at heterogeneous surfaces: molecular-dynamics simulations and hydrodynamic description. Eur Phys J E 15:427-438

Cottin-Bizonne C, Steinberger A et al (2008) Nanohydrodynamics: the intrinsic flow boundary condition on smooth surfaces. Langmuir 24:1165-1172

Craig VSJ, Neto C et al (2001) Shear-dependent boundary slip in an aqueous Newtonian liquid. Phys Rev Lett 87:054504

Donose BC, Vakarelski IU et al (2005) Silica surfaces lubrication by hydrated cations adsorption from electrolyte solutions. Langmuir 21:1834-1839

Du Buat PLG (1779) Principes d'Hydraulique. L'imprimerie de monsieur, Paris

Ducker WA, Senden TJ et al (1991) Direct measurement of colloidal forces using an atomic force microscope. Nature 353:239-241

Ellis JS, Thompson M (2004) Slip and coupling phenomena at the liquid-solid interface. Phys Chem Chem Phys 6:4928-4938

Fan TH, Vinogradova OI (2005) Hydrodynamic resistance of closeapproached slip surfaces with a nanoasperity or an entrapped nanobubble. Phys Rev E 72:066306

Guriyanova S, Bonaccurso E (2008) Influence of wettability and surface charge on the interaction between an aqueous electrolyte solution and a solid surface. Phys Chem Chem Phys 10:4871-4878

Hocking LM (1976) Moving fluid interface on a rough surface. J Fluid Mech 76:801-817

Honig C, Ducker W (2007) Thin film lubrication for large colloidal particles: experimental test of the no-slip boundary condition. J Phys Chem C 111:16300-16312

Honig C, Ducker W (2008) Squeeze film lubrication in silicone oil: experimental test of the no-slip boundary condition at solidliquid interfaces. J Phys Chem C 112:17324-17330

Hutter JL, Bechhoefer J (1993) Calibration of atomic force microscope tips. Rev Sci Instrum 64:1868-1873

Jabbarzadeh A, Atkinson JD et al (2000) Effect of wall roughness on slip and rheological properties of hexadecane in molecular dynamics simulation of Couette shear flow between two sinusoidal walls. Phys Rev E 61:690-699

Joseph P, Tabeling P (2005) Direct measurement of the apparent slip length. Phys Rev E 71:035303

Lauga E, Brenner MP et al (2005) Microfluidics: the no-slip boundary condition. Springer, New York

Lee C, Choi CH et al (2008) Structured surfaces for a giant liquid slip. Phys Rev Lett 101:064501

Lukyanov AV (2009) Liquid flow over a substrate structured by seeded nanoparticles. Phys Lett A 373:1967-1971

Maali A, Cohen-Bouhacina T et al (2008) Measurement of the slip length of water flow on graphite surface. Appl Phys Lett 92: 053101
Matei GA, Thoreson EJ et al (2006) Precision and accuracy of thermal calibration of atomic force microscopy cantilevers. Rev Sci Instrum 77:083703

Neto C, Craig VSJ (2001) Colloid probe characterization: radius and roughness determination. Langmuir 17:2097-2099

Neto C, Evans DR et al (2005) Boundary slip in Newtonian liquids: a review of experimental studies. Rep Prog Phys 68:2859-2897

Ohler B (2007) Cantilever spring constant calibration using laser Doppler vibrometry. Rev Sci Instrum 78:063701

Pit R, Hervet $\mathrm{H}$ et al (2000) Direct experimental evidence of slip in hexadecane: solid interfaces. Phys Rev Lett 85:980-983

Ponomarev IV, Meyerovich AE (2003) Surface roughness and effective stick-slip motion. Phys Rev E 67:026302

Prandtl L (1927) Motion of fluids with very little viscosity. National Advisory Committee for Aeronautics: Technical memorandum No 452

Qian TZ, Wang XP et al (2005) Hydrodynamic slip boundary condition at chemically patterned surfaces: a continuum deduction from molecular dynamics. Phys Rev E 72:022501

Richardson S (1973) No-slip boundary-condition. J Fluid Mech 59:707-719

Schmatko T, Hervet $\mathrm{H}$ et al (2006) Effect of nanometric-scale roughness on slip at the wall of simple fluids. Langmuir 22:6843-6850

Stark R, Bonaccurso E et al (2006) Quasi-static and hydrodynamic interaction between solid surfaces in polyisoprene studied by atomic force microscopy. Polymer 47:7259-7270

Stokes GG (1845) On the theories of the internal friction of fluids in motion. Trans Cambridge Philos Soc 8:287-315

Stokes GG (1966) Mathematical and physical papers by George Gabriel Stokes, vol 1. Cambridge University Press, Cambridge, pp 75-187

Sun G, Bonaccurso E et al (2002) Confined liquid: simultaneous observation of a molecularly layered structure and hydrodynamic slip. J Chem Phys 117:10311-10314

Tretheway DC, Meinhart CD (2002) Apparent fluid slip at hydrophobic microchannel walls. Phys Fluids 14:L9-L12

Tretheway DC, Meinhart CD (2004) A generating mechanism for apparent fluid slip in hydrophobic microchannels. Phys Fluids 16:1509-1515

Truesdell R, Mammoli A et al (2006) Drag reduction on a patterned superhydrophobic surface. Phys Rev Lett 97:044504

Vinogradova OI (1995) Drainage of a thin liquid film confined between hydrophobic surfaces. Langmuir 11:2213-2220

Vinogradova OI (1999) Slippage of water over hydrophobic surfaces. Int J Mineral Proc 56:31-60

Vinogradova OI, Yakubov GE (2006) Surface roughness and hydrodynamic boundary conditions. Phys Rev E 73:045302

Vinogradova OI, Butt H-J et al (2001) Dynamic effects on force measurements. 1. Viscous drag on the atomic force microscope cantilever. Rev Sci Instrum 72:2330-2339

Willmott GR, Tallon JL (2007) Measurement of Newtonian fluid slip using a torsional ultrasonic oscillator. Phys Rev E 76:066306

Willmott GR, Tallon JL (2008) Nanoscale slip measurements using a torsional ultrasonic oscillator. Curr Appl Phys 8:433-435

Zhu Y, Granick S (2001) Rate-dependent slip of Newtonian liquid at smooth surfaces. Phys Rev Lett 87:096105

Zhu YX, Granick S (2002) Limits of the hydrodynamic no-slip boundary condition. Phys Rev Lett 88:096102

Zhu Y, Granick S (2004) Superlubricity: a paradox about confined liquids resolved. Phys Rev Lett 93:096101 